\title{
Research of friction characteristics on the hydraulic transportation in inclined slurry-pipe
}

\author{
Wang Tieli ${ }^{1}$ \\ ${ }^{1}$ Wuhan Design and Research Institute Co., Ltd of China Coal Technology Engineering Group, Wu Han, Hubei \\ Province ,430064, China
}

\begin{abstract}
By analyzing the momentum transfer and velocity both of solid particles and water over the acceleration time of solid particles, as well as interaction mechanism between water and solid particals, a new model is proposed to predict friction loss for setting slurry flow in inclined pipe. The hydraulic gradient formula for inclined pipes summarized by the author is confirmed by a large amount of experimental data. The results show that the deviation between the theoretical value of the model proposed by the author and the measured value is not more than $13.33 \%$, which is the smallest among all reports.
\end{abstract}

\section{Introduction}

With the increasing demand of power plants and chemical companies for mineral resources and the increasingly stringent requirements for the environment, pipeline transportation of coal slurry is more and more widely used due to its economy and environmental friendliness. Generally, the hydraulic gradient of settling slurry in the inclined pipe needs to be calculated to judge the stability of long-distance slurry transportation. However, due to its complexity, there are few reports in this area at present. Rose [1], Kawashima [2] and Worster [3] have studied many transportation parameters in inclined pipelines, but the research on hydraulic gradient and inclined slurry flow characteristics is still incomplete.

Generally, the pressure loss of the slurry directly depends on the state of motion of the solid particles. Based on a new theoretical analysis method[4], a new model is proposed to reveal the relationship between the movement status of solid particles and the pressure loss of setting slurry. In this model, the hydraulic gradient of setting slurry in different motion states (in suspension, by saltation, or in partly suspension and by saltation) in inclined pipe can be predicted clearly.

\section{The velocity change of the water during solid particles' accelerating}

Using a new theoretical analysis method proposed by the author, it is possible to set the momentum of the solid particles with mass Ms and the water with mass Mw from the pipe outlet $\mathrm{A}$ at time $\mathrm{t}$ and the balance equation of the continuous equation. And get the following equation [4]:

$$
\begin{aligned}
& V-V_{\mathrm{w}}=\frac{k_{2}^{2} \overline{\mathrm{q}} \rho_{\mathrm{s}} V_{\mathrm{s}}^{2}}{k_{1}(1-\overline{\mathrm{q}}) \rho V_{\mathrm{w}}} \\
& V_{\mathrm{m}}=V_{\mathrm{w}}(1-\overline{\mathrm{q}})+V_{\mathrm{s}} \overline{\mathrm{q}}
\end{aligned}
$$

where and $V$ is the average rate of the water before momentum transference. $\rho$ and $\rho_{\mathrm{s}}$ are the density of water and solid particles. q, $V_{\mathrm{w}}, V_{\mathrm{s}}, V_{\mathrm{m}}$ are separately in-situ average concentration (by volume) of solid particles, the average velocity of the water and the solid particles and settling slurry in the section $A . k_{1}$ and $k_{2}$ are coefficient. And the equation to calculate $k_{1}, k_{2}$ are:

$$
\begin{aligned}
& k_{1}=1 /[1-0.56 \psi(1) \varphi(\theta)] \quad(3) \\
& k_{2}=1+k_{4}(33 \lambda \cos \theta \pm \sin \theta) \frac{k_{3} \sqrt{L_{a} g}}{V_{m}}\left(1-\frac{1}{\delta}\right)
\end{aligned}
$$

It can be seen from Rose's experimental results that the value of $\psi(1)$ is closely related to the average velocity $V_{m}$ of the two-phase flow in the pipeline, the particle size $d$ and the density ratio $\delta$ of solid particles to the fluid. According to the experimental data of several scholars, the fitting curve between $\psi(1)$ and $\log _{10}$ $\left(V_{m}{ }^{2} /\left(\mathrm{g} d \delta^{2}\right)\right.$ is shown in Figure 1[5]. According to Rose's report[1], $\varphi(\theta)$ is a function of $\theta$ in an up-inclined pipe (Figure 2), but in a down-inclined pipe, $\varphi(\theta)$ is smaller than its value in an up-incline pipe. Here we assume that $\varphi(\theta)$ in the up-incline pipe and the down-inclined pipe are symmetric (Figure 3), so that $\varphi(\theta)$ in the down-inclined pipe can be predicted.

*Corresponding author's e-mail: wuhanwangtieli@163.com 


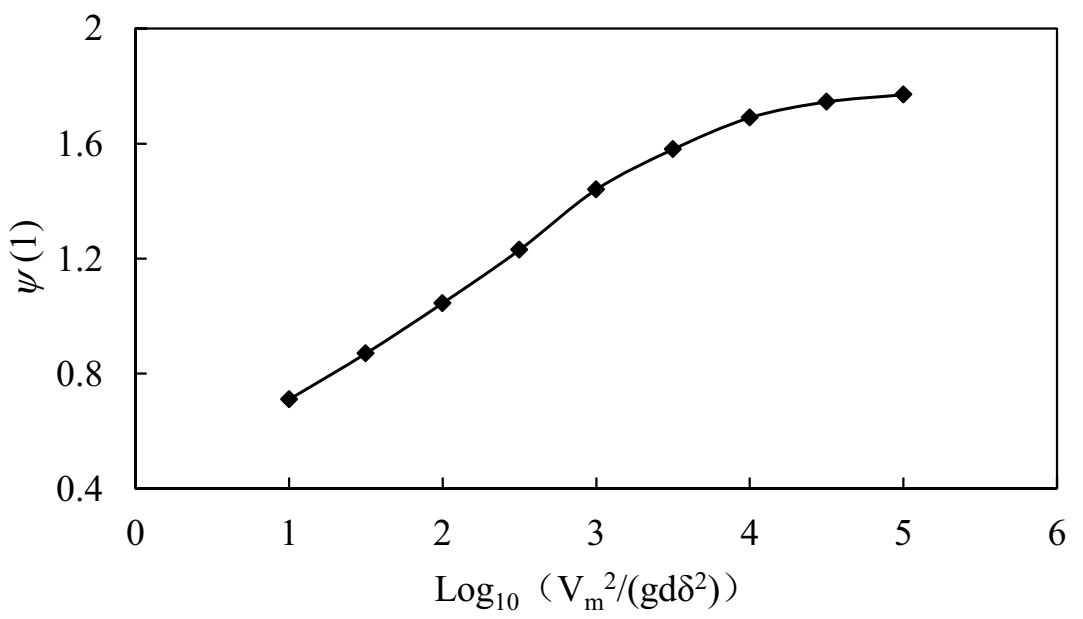

Figure 1. Relationship of $\psi(1)$ with $\mathrm{V}_{\mathrm{m}}^{2} / \mathrm{gd} \delta^{2}$

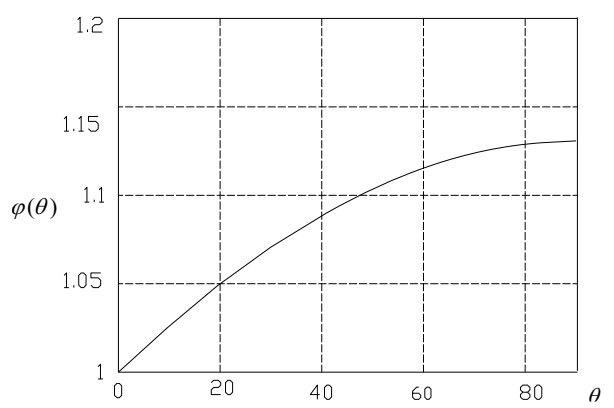

Figure 2. The relationship between $\varphi(\theta)$ and $\theta$ for up-inclined pipe
In equation (4), $\delta=\rho_{s} / \rho, k_{3}=3.742, \lambda$ is friction factor. When solid particles are transported in a suspended state, $\mathrm{k}_{4}$ represents the proportional coefficient of the solid particles in the sliding state to the total weight of the solid particles, so when all solid particles are suspended, $\mathrm{k}_{4}=0$; when all solid particles are transported in a saltation state, $\mathrm{k}_{4}=1$; When part of the solid particles are transported in a suspended state and another part is transported in a saltation state, $k_{4}=V_{\mathrm{t}} / V_{\mathrm{m}}$, where $V_{\mathrm{m}}$ is the average velocity of the condensed slurry, $V_{\mathrm{t}}$ is the final velocity of the particles, and $\mathrm{L}_{\mathrm{a}}$ is the length of the pipe required to fully accelerate the solid, the calculation formula is as follows:

$\mathrm{L}_{\mathrm{a}}=6 \mathrm{D}\left(\frac{\mathrm{M}_{\mathrm{s}}}{\rho \sqrt{\mathrm{g}} \sqrt{\mathrm{D}^{5}}} \sqrt{\frac{\mathrm{D}}{\mathrm{d}}} \frac{\rho_{\mathrm{s}}}{\rho}\right)^{\frac{1}{3}}$

Where $\mathrm{D}$ represents the diameter of the pipe, $\mathrm{d}$ stands

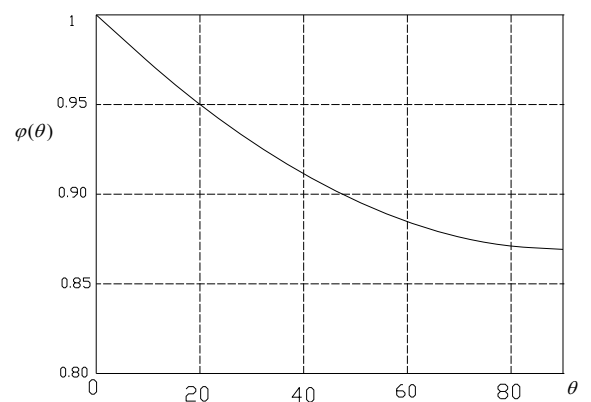

Figure 3. The relationship between $\varphi(\theta)$ and $\theta$ for down-inclined pipe

for the diameter of the solid particles, $\mathrm{M}_{\mathrm{s}}$ is the mass flow rate of the solid, and $\mathrm{g}$ stands for the acceleration due to gravity [1].

\section{Analysis of the forces that the solid particles exerted}

As shown in Figure 4, when the slurry flows in the inclined pipe, the solid particles are subjected to four forces, which are the drag force of the fluid on the particles $\left(F_{D}\right)$, the gravity sliding component of the solid particles $\left(W_{b} \sin (\theta)\right)$, and the interference forces of other particles $\left(F_{h}\right)$ and the friction against the particles $\left(K_{4} f_{f}\right)$. 


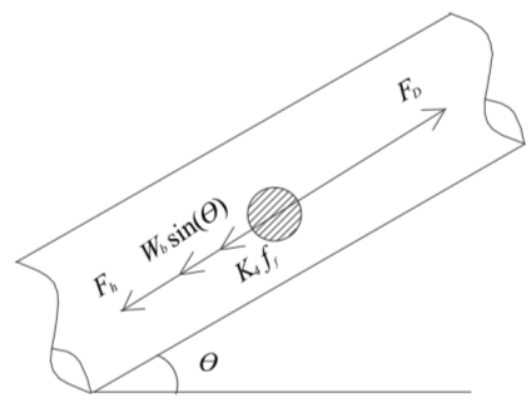

up-inclined pipe

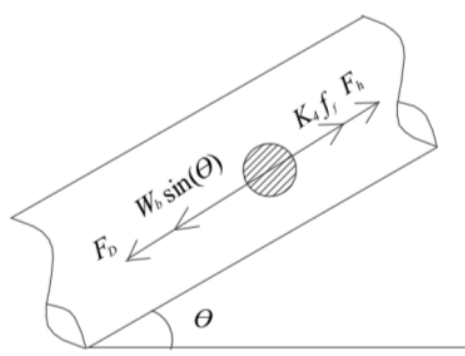

down-inclined pipe

Figure 4. Diagram of force acting on particles in inclined pipeline

The equation for the acceleration of a solid is as follows:

$\frac{\pi}{6} d_{e}^{3}\left(\rho_{s}+\frac{\rho}{2}\right) \frac{d V_{s}}{d t}=F_{D}-F_{h}-k_{4} f_{f} \mp W_{b} \sin \theta$

where

$F_{\mathrm{D}}=\frac{\pi}{4} \mathrm{~d}^{2} \mathrm{C}_{\mathrm{D}} \frac{\left(V_{\mathrm{w}}-V_{\mathrm{s}}\right)^{2}}{2} \rho$

$F_{\mathrm{b}}=\left(1-(1-\overline{\mathrm{q}})^{2(\mathrm{n}-1)} \frac{\left(\sqrt{\operatorname{Rep}} \alpha+\sqrt{\operatorname{Rep} \alpha^{2}+4 \sqrt{48} \alpha \beta /(1-\overline{\mathrm{q}})^{\mathrm{n}-1}}\right)^{2}}{\left(\sqrt{\operatorname{Rep} \alpha}+\sqrt{\operatorname{Rep} \alpha^{2}+4 \sqrt{48} \alpha \beta}\right)^{2}} W_{\mathrm{b}}\right.$

$f_{f}=\frac{\pi}{c} \mathrm{~d}^{3} \lambda\left(\rho_{\mathrm{s}}-\rho\right) \mathrm{g} \cos \theta$

and $F_{D}$ represents the drag force of water, $F_{h}$ stands for the interference force from other solid particles, $f_{f}$ is the friction force acting on a single solid particle, $\mathrm{d}$ is the diameter of the solid particle, $\mathrm{w}_{\mathrm{b}}$ is the effective gravity of the solid particle in water, and its expression is: $W_{\mathrm{b}}=\pi \mathrm{d}^{3}\left(\rho_{\mathrm{s}}-\rho\right) \mathrm{g} / \mathrm{c} ;$ In addition, $\mathrm{C}_{\mathrm{D}}$ is the drag coefficient calculated from $V_{\mathrm{w}}-V_{\mathrm{s}}, R_{e p}$ is the Reynolds factor of solid particles, and $\alpha, \beta$ are Swanson shape factors. $\mathrm{n}$ is a prime number calculated using the Sato Hiroshi equation. After the solid particles are accelerated, the setting slurry will become a stable flow state, and the velocities of water, solid particles and slurry will also become the same. At this time, the acceleration of the solid particle $d V_{s} / d_{t}=0$, so the equation (6) becomes as follows:

$$
F_{D}-F_{h}-k_{4} f_{f} \mp W_{b} \sin (\theta)=0
$$

When the fluid moves in the upward inclined pipe, $W_{b} \sin (\theta)$ is a negative value, otherwise it is a positive value.

Substituting equation (7), (8) into equation (10) and combining equation (9) to obtain equation (11) as follows:
$V_{s}=V_{W}-\sqrt{\frac{8\left(F_{h}+k_{4} f_{f}\right) \mp W_{b} \sin (\theta)}{\pi d_{e}^{2} C_{D r} \rho}}$

Substituting equation (3) into equation (1) and combing equation (4) to obtain equation (12) as follows:

$V=V_{s}+\left[1-0.5 \zeta_{s} \psi(1) \varphi(\theta)\right] \frac{\delta \bar{q}}{(1-\bar{q}) V_{v}} V_{s}^{2}\left[1+k_{4}(33 \lambda \cos (\theta) \pm \sin (\theta)) \frac{k_{s} \sqrt{L_{s} g}}{V_{s}}\left(1-\frac{1}{\delta}\right)\right]^{2}$

\section{The hydraulic gradient of setting slurry}

The hydraulic gradient when the water is in a turbulent state can be calculated by the following equation:

$i=\frac{\lambda}{2 g D} V^{2}$

where $D$ is the inner diameter of the pipe, $V$ stands for the average velocity of the water. Obviously, when the setting slurry moves in a suspended state, a jumping state or a partly jumping and partly suspended state, equation (13) cannot be used to calculate the hydraulic slope. However, it can be seen from equation (12) that the setting slurry flowing at an average velocity $V_{\mathrm{m}}$ can be regarded as water flowing at an average velocity $V$. More specifically, if the pressure difference existing in the pipe section can make water move forward at a velocity $V$, then after being intervened by group of solid particles with the average volume concentration $\overline{\mathrm{q}}$, the pressure difference can only make the mixture (setting slurry) move forward with the velocity $V_{m}$. Therefore, the hydraulic gradient generated when setting slurry flowing at the velocity $V_{m}$ flows in the pipeline is equivalent to the hydraulic gradient of the water flowing at the velocity $V$ in the same pipeline. The equivalent resistance model in Figure 5 can illustrate the above point very well. Therefore, only when the water velocity $V$ in the pipeline can be calculated, the hydraulic gradient of the setting slurry can be obtained. 


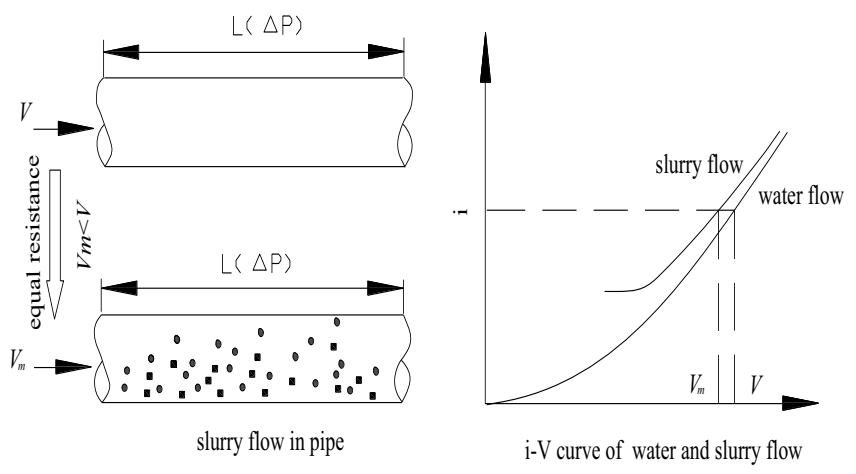

Figure 5. Equivalent Resistance model

According to the previous analysis results, the equation to calculate hydraulic gradient of the setting

slurry can be obtained as follows:

$$
i=\frac{\lambda}{2 g D}\left\{V_{w}+[1-0.56 \psi(1) \varphi(\theta)] \frac{\delta \bar{q}}{(1-\bar{q}) V_{w}} V_{s}^{2}\left[1+k_{4}(33 \lambda \cos \theta \pm \sin \theta) \frac{k_{3} \sqrt{L_{a} g}}{V_{m}}\left(1-\frac{1}{\delta}\right)\right]^{2}\right\}^{2}
$$

\section{The criterion of the solid particles' moving status}

As is mentioned above, $k_{4}$ represents the proportion coefficient of solid particles in the slip state to the total weight of solid particles, Its value is mainly related to the state of particle movement [6]. When the average velocity of the setting slurry exceeds the limit flow velocity $\left(V_{H}\right)$ of the homogeneous fluid, it can be considered that the particles are transported in a suspended state. When average velocity of setting slurry is between the deposit limit velocity $V_{c d}$ and the float limit velocity $V_{B}$, solid particles can be considered as being transported by saltation. When average velocity of setting slurry is between the float limit velocity $V_{B}$ and the homogeneous limit velocity $V_{H}$, solid particles can be considered as being transported in suspension partly and by saltation partly. When the diameter of the solid particles is evenly distributed, it is easy to judge the state of movement. However, when the distribution of the solid particle diameter is within a certain range, it is difficult to determine its motion state. As to the setting slurry when the diameter of the solid particles are rather single, the floating limit velocity $V_{B}$ and the homogeneous limit velocity $V_{H}$ can be calculated with the following Newitt and Lazarus' equations [7]:

$$
\begin{gathered}
\mathrm{V}_{\mathrm{B}}=17 \mathrm{~V}_{\mathrm{t}} \\
\mathrm{V}_{\mathrm{H}}=4.4 \sqrt{\mathrm{g} \mathrm{D}} \mathrm{C}_{\mathrm{D}}^{-0.25}(\delta-1)^{\frac{1}{\zeta}}
\end{gathered}
$$

\section{The comparison of the theoretical results with experimental results}

So far, several experts have proposed their calculation models for the friction loss of inclined pipe slurry as follows:

Kawashima's model can be expressed in following:
$\frac{i-i_{W}}{i_{W} C_{V}}= \pm \frac{3}{2} \frac{C_{D}}{\lambda}\left(\frac{d}{D}\right)^{-1} \frac{(1-\zeta)^{2}}{\zeta}$

Where $i_{w}$ stands for hydraulic gradient of water, $C_{V}$ represents slurry volume concentration, $\zeta$ is a variable related to particle size $d$, specific gravity $\delta$, and pipe inclination $\theta[2]$.

When $\mu_{s} \cos \theta+\sin \theta>0$, the right sign of equation (17) is positive, and the expression of $\zeta$ is as follows:

$$
\bar{\xi}=1+\frac{1}{V_{m}} \sqrt{\frac{4(\rho-1) g d}{3 C_{\mathrm{D}}}\left(\mu_{s} \cos \theta+\sin \theta\right)}
$$

When $\mu_{s} \cos \theta+\sin \theta<0$, the right sign of equation (17) is negative, and the expression of $\zeta$ is as follows:

$$
\bar{\xi}=1-\frac{1}{V_{m}} \sqrt{\frac{4(\rho-1) g d}{3 C_{\mathrm{D}}}\left(\mu_{s} \cos \theta+\sin \theta\right)}
$$

When $\mu_{s} \cos \theta+\sin \theta=0, \quad \zeta=1, \quad i=i_{w}$

Where $u_{s}$ is friction coefficient

Worster's model[2] can be expressed in following:

$$
i_{m}=i_{0}+\left(i_{h}-i_{0}\right) \cos (\theta) \pm C_{V}(\delta-1) \sin (\theta)
$$

Where $i_{h}$ stands for the hydraulic gradient of slurry in horizontal pipeline with the same concentration.

In Figure 6, Figure 7 and Figure 8, Huang zhao-lin's experiment data (down inclined) has been used to verify theoretical model[8]. In Huang zhao-lin's experiment, solid particle diameter is $0.2042 \mathrm{~mm}$, and relative density is 1.552 . The slurry volume concentration is $21.74 \%$. In Figure 6, pipe inclination is 15 degree, pipe inside diameter is $20 \mathrm{~mm}$. In Fig.7, pipe inclination is 30 degree, pipe inside diameter is $15 \mathrm{~mm}$. In Figure 8, pipe inclination is 40 degree, pipe inside diameter is $15 \mathrm{~mm}$. 
In figure 6 , the maximum deviation between the author formula calculating values and measured values is $9.71 \%$, and the value is $11.09 \%, 52.91 \%$ for Worster and Kawashima formula respectively.

Some important information can be obtained from Figure 7 , the maximum deviation between the author formula calculating values and measured values is $10.33 \%$, and $63.44 \%, 7.07 \%$ for Worster and Kawashima formula respectively. Truthfully speaking, the calculation deviation of the author's formula is not obvious.

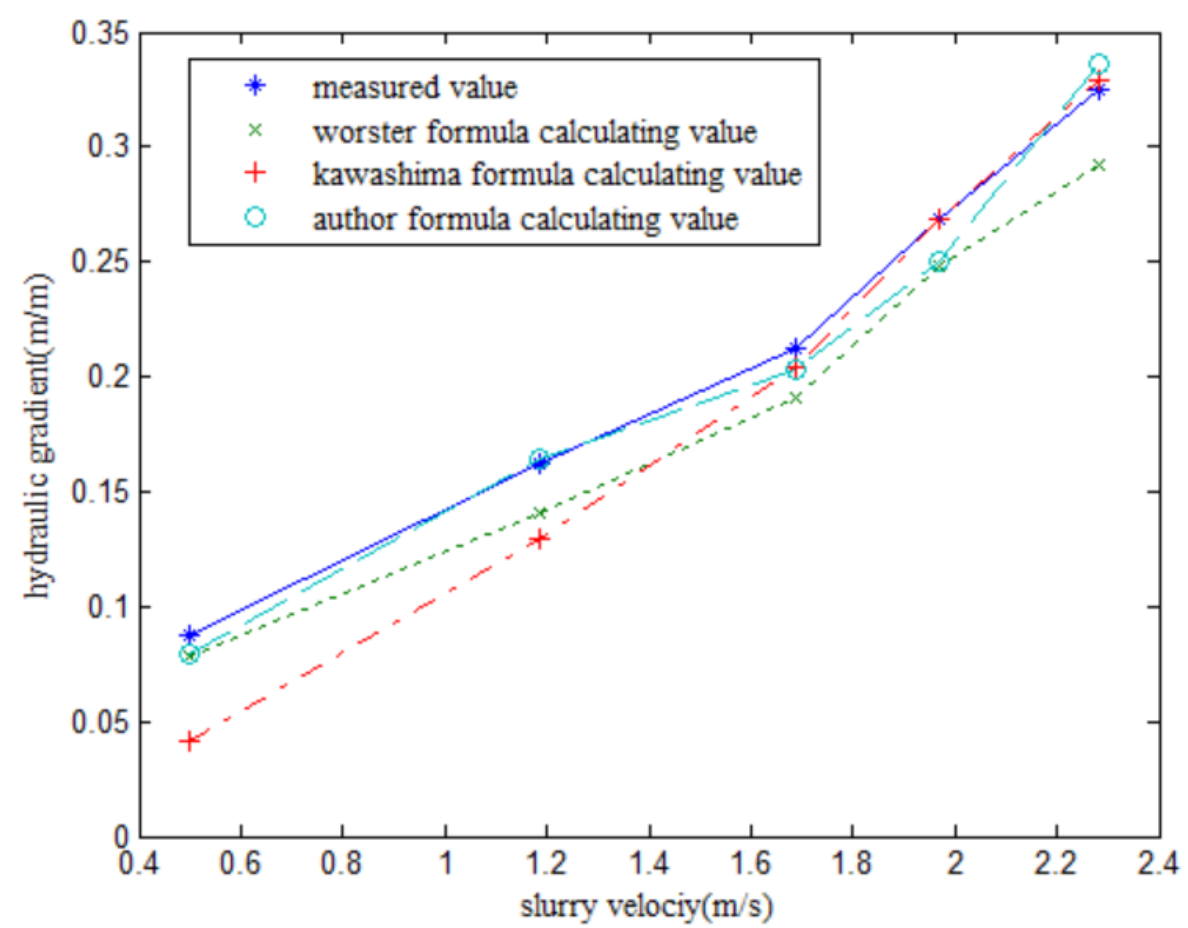

Figure 6. Comparison of Several Calculating Formulas for $D=20 \mathrm{~mm}, \theta=15^{\circ}$

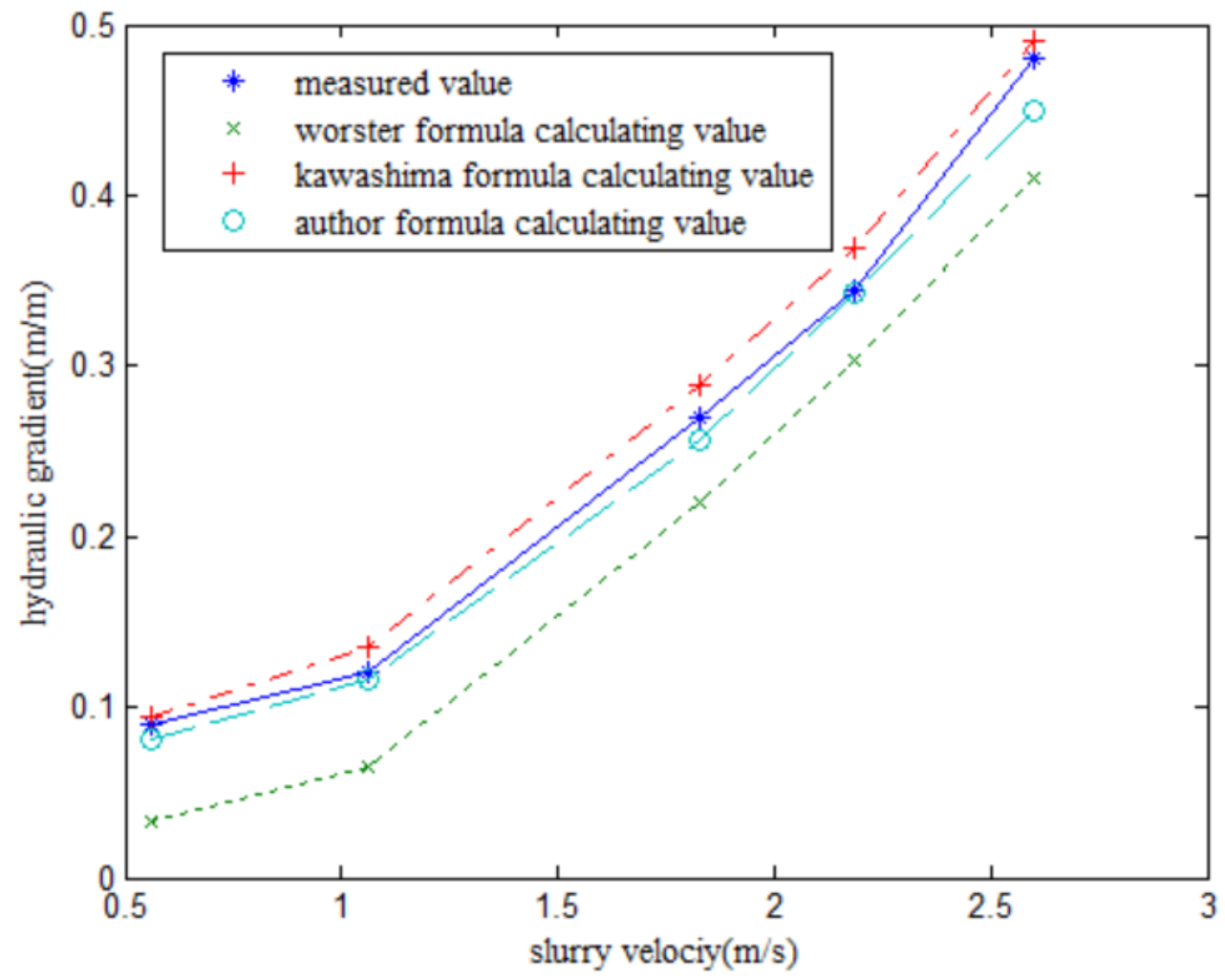

Figure 7. Comparison of Several Calculating Formulas for $D=15 \mathrm{~mm}, \theta=30^{\circ}$ 


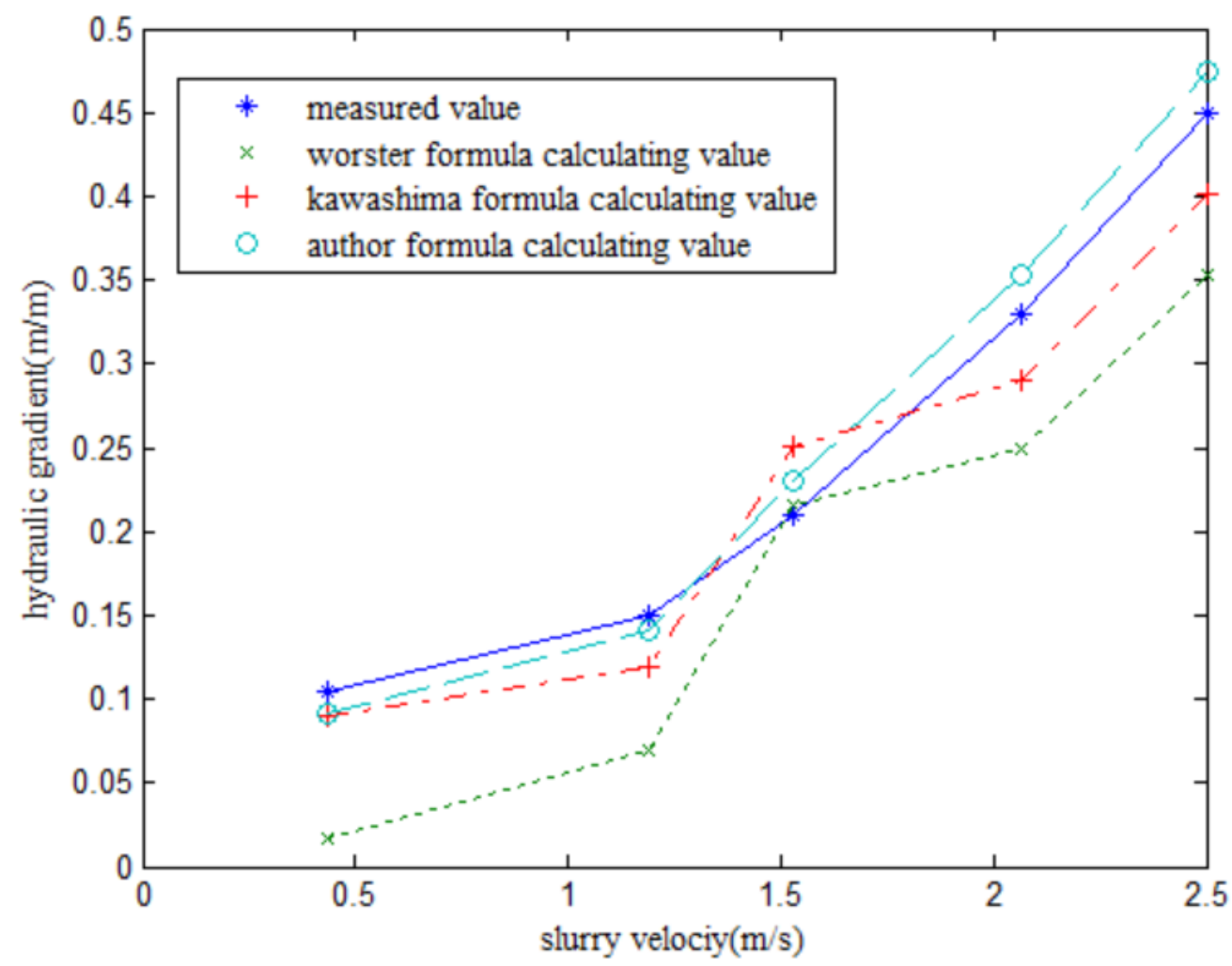

Figure 8. Comparison of Several Calculating Formulas for $D=15 \mathrm{~mm}, \theta=40^{\circ}$

In figure 8 , the maximum deviation between the author formula calculating values and measured values is $13.33 \%$, and $84.29 \%, 20.93 \%$ for Worster's and Kawashima's formula respectively. Generally speaking, the calculation deviation of the author's formula is small.

Except for individual data points, the deviation calculated by the author's formula is the smallest from Figure 6, Figure 7 and Figure 8.

In Figure 9 and Figure 10, Diniz and Coiado[9] inclined experiment results(up inclined) and detailed experiment procedure are investigatived. In his experiment, solids diameter is $0.20 \mathrm{~mm}$, pipe inside diameter is $75 \mathrm{~mm}$ and relative density value is 2.68 . The slurry volume concentration is $5 \%$. In Figure 9 and Figure 10, pipe inclination is 11 and 34 degree, respectively.

In Figure 9, the maximum deviation between the author formula calculating values and measured values is $8.71 \%, 14.21 \%$ and $42.36 \%$ for Worster's model and Kawashima's formula respectively. In Figure 10, the maximum deviation between the author formula calculating values and measured values is $5.79 \%, 7.47 \%$ and $22.84 \%$ for Worster's model and Kawashima's formula respectively. It is clear that in Figure 9 and Figure 10, the deviation of the author's formula is the smallest. 


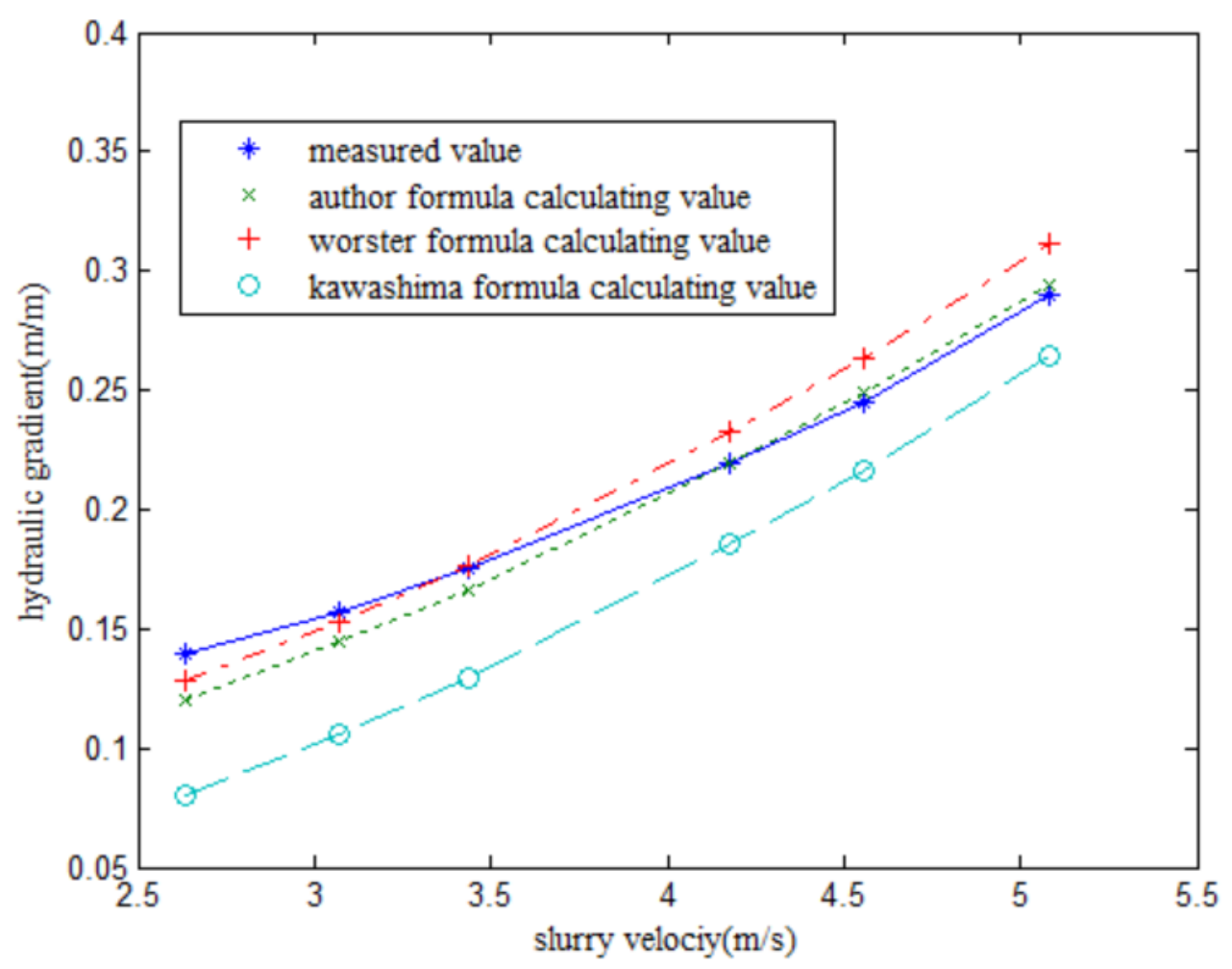

Figure 9. Comparison of several calculating formulas

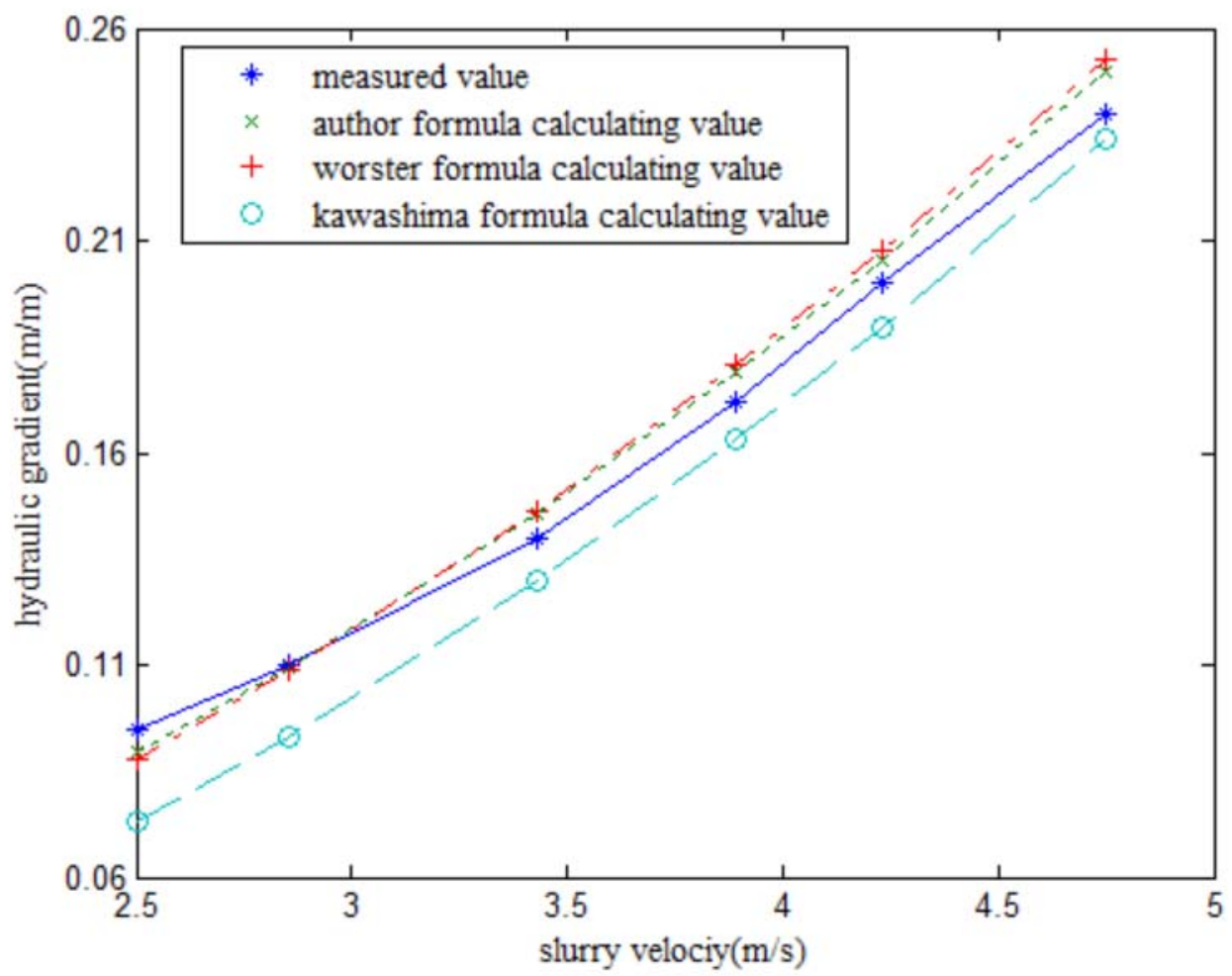

Figure 10. Comparison of Several Calculating Formulas

From Fig.6-10, the author's model is consistent with measured values and the deviation is not exceed $13.33 \%$, while the other two models have larger deviation. It can be concluded that when the slurry velocity is low, all the three model's calculating deviation is large. From the deduction of
Kawashima's model, there is not used for solid in suspending state. As for low velocity, the deviation of Kawashima's model is mainly that value of coefficient of pressure loss is not suitable for medium particles. For Worster's model, reason of deviation is probably due to its less influencing 
factors considered.

In Figure 6, experiment data just for down inclined flow is given out, in order to facilitate comparison and analysis, the upward inclined flow data and the horizontal flow data under the condition of Fig. 6 are also presented in Table 1.

Table 1. Comparison and Analysis of Slurry Inclined Flow and Horizontal Flow

\begin{tabular}{cccccc}
\hline \multirow{2}{*}{$\begin{array}{c}\text { Slurry average } \\
V_{m} /(\mathrm{m} / \mathrm{s})\end{array}$} & \multicolumn{4}{c}{ hydraulic gradient $i /(\mathrm{m} / \mathrm{m})$} \\
\cline { 2 - 6 } & measured value & Author formula & Kawashima & Worster & morizontal pipe \\
\hline 0.5 & $0.1375(0.0875)$ & $0.1375(0.079)$ & 0412 & $0.1340(0.0778)$ & 0.0145 \\
1.1875 & $0.1938(0.1625)$ & $0.1874(0.1639)$ & 0.1295 & $0.2027(0.1405)$ & 0.0820 \\
1.6875 & $0.2375(0.2125)$ & $0.2312(0.2032)$ & 0.2038 & $0.2535(0.1913)$ & 0.1657 \\
1.9688 & $0.2813(0.2688)$ & $0.2988(0.2501)$ & 0.2685 & $0.3101(0.2479)$ & 0.2255 \\
2.2813 & $0.3375(0.325)$ & $0.3434(0.3358)$ & 0.3289 & $0.3544(0.2922)$ & 0.3028 \\
\hline
\end{tabular}

It can be discovered that from Huang zhao-lin's experiment, when the pipe inclination is 15 degree and -15 degree, hydraulic gradient values are completely different. The maximum deviation between the author formula calculating values and measured values is $6.22 \%$, and $70 \%, 10.24 \%$ for Kawashima and Worster formula for up-inclined flow, respectively.

According to Kawashima formula calculated result, $\mu_{s} \cos \theta+\sin \theta<0$, hydraulic gradient values of up-inclined and down-inclined are identical, the situation seems unreasonable, it can be seen from Figure 6 Figure 10 and table 1 that there is a large deviation existing between the calculated values of Kawashima formula and the measured values.

Solid diameter and friction factor have not appeared in Worster's formula (20), but the hydraulic gradient in inclined pipe can be affected by these two factor. So Worster's formula is imperfect, especially for the slurry flow at low speed. As for author's model, possible reason is that the parameter $k_{4}$ is not properly determined when flow velocity is low. It is obvious that when slurry velocity is very low, the flow of solid is quit complicated. So discuss the parameter $k_{4}$ when slurry velocity at very low level is really essential.

\section{Conclusion}

By analyzing the change of solid particles and water over the acceleration time of solid particles in momentum, velocity and interplay mechanism of water and solid particles, a new model that can employed to calculate the hydraulic gradient of settling slurry in inclined pipe is put forward in the paper. The proposed new model was verified on the basis of experimental data from several experts, and the calculated values were not much deviation compared with the experimental values. So when solid particle diameter is about $0.2 \mathrm{~mm}$, pipe diameter is in the range of $15-75 \mathrm{~mm}$, and inclination angle is in the range of 10-40 degree. We get reason to believe the author's model is superior to Kawashima and Worster's model. As to other case, more research has to be done.

\section{References}

1. Rose, H. E. and Duckworth, R. A. (1969) Transport of solid particles in liquids and gases. THE ENGINEER, 226 (12): 430- 434.

2. Hiroshi S, (1995) Transportation System, Akita Typography Co., Ltd. 86 164 .

3. Durand R. (1952) The hydraulic transportion of coal and solid materials in pipes colloq of National coal Board, London. $39 \sim 52$.

4. Xu Z. (1998) The study of the velocity profile for settling slurry flow in a horizontal pipe. Journal of Liaoning technical university, 17(5): 449-454.

5. Xu Z. (1998) The study of the mechanism of settling slurry flow in a horizontal pipe. Journal of Liaoning technical university, 17(2): 113-118.

6. Zhao L. (2011) Study on Flow Law of Large particle slurry in pipeline, Liaoning Technical University.

7. Xu Z. (1999) The Study of Velocity Profile and Hydraulic Gradient of Settling Slurry, Northeast University.

8. Huang Z, (1985) Experimental study of the characteristic of flows slurry in inclined pipe, Experimental study of the characteristic that the thick liquid body flows in the pipeline that slope , master thesis of Chinese mining institute.

9. Diniz and Coiado. (1991)Two - Phase ( solid liquid ) flow In Inclined pipes, BHR Group, Hydrotransport 14. 